\title{
Ultrasonographic characteristics and outcome of Type III umbilical-portal-systemic venous shunt
}

\author{
Linlin Zhu, Haifang Wu, Xiang Cong, Zhe Ma, Guowei Tao
}

Department of Ultrasound, Qilu Hospital of Shandong University, Jinan, Shandong Province, China

\begin{abstract}
Aims: According to a novel in-utero classification termed "umbilical-portal-systemic venous shunt (UPSVS)" recently proposed for an abnormal umbilical, portal and ductal venous system, the portal-systemic shunt belongs to type III UPSVS. This study was designed to examine the ultrasonographic characteristics and outcome of type III UPSVS. Material and methods: All cases of Type III UPSVS diagnosed at our department from April 2016 to December 2020 were retrospectively studied. Results: Seventeen patients with type III UPSVS including 12 type IIIa and 5 IIIb cases were identified. Sonography showed a shunt between the inferior left portal vein and the left hepatic vein in all type IIIa cases. Three cases of type IIIb had a combination of another shunt ( 2 with type I and one with type IIIa). Integrate intrahepatic portal vein system was not seen in those 2 cases of type IIIb combined with type I UPSVS, leading to termination of pregnancy (TOP). TOP occurred in 4 patients with type IIIa as requested by the parents. Two cases (type IIIa and type IIIb each) underwent surgical procedure for the closure of the shunt. Spontaneous complete closure in 4 type IIIa cases and partial closure in one type IIIb case occurred during a period of 3-16 months. Conclusions: The majority of patients had type IIIa UPSVS presenting a good outcome. The lack of integrate intrahepatic portal vein system was the main reason for TOP in patients with type IIIb UPSVS. These data suggest the UPSVS classification is a useful tool for a prognosis prediction of type III UPSVS.

Keywords: ultrasonography; umbilical-portal-systemic venous shunt; portosystemic shunt; termination of pregnancy; prognosis prediction
\end{abstract}

\section{Introduction}

An abnormal communication between the portal vein and the systemic venous system causes the portosystemic shunt (PSS), resulting in complete or partial diversion of the portal flow from the liver to the systemic venous circulation [1]. This condition, also termed as "congenital portosystemic shunt", has been described mostly in the pediatric population [1-3]. With the development of prenatal screening techniques, especially ultrasonography [4], diagnosis of fetal PSS has been described in

Received 19.03.2021 Accepted 06.06.2021

Med Ultrason

2022, Vol. 24, No 1, 14-18

Corresponding author: Prof. Guowei Tao

Department of Ultrasound,

Qilu Hospital of Shandong University, 1

07 Wenhua West Road, Jinan 250012, China

E-mail: taoguowei@yahoo.com

Phone: 0086-531-82169114 case studies [5-8]. Nevertheless, the manifestations and evolution of fetal PSS, which are important for prenatal counselling and perinatal management, are still poorly understood [9].

The umbilical vein (UV), portal vein (PV) and ductus venosus (DV) in fetus form an integral conduit, through which the highly oxygenated blood is transported from the placenta into the fetal heart [10]. Malformation of $\mathrm{UV}, \mathrm{PV}$ and DV can cause shunting into the systemic circulation. In 2016, Achiron and Kivilevitch defined the "umbilical-portal-systemic venous shunt (UPSVS)" and proposed a novel classification of in-utero UV-PV-DV anomalies [11]. According to the anatomical origin of the shunt (umbilical, portal or ductal), the UPSVS is classified into 4 types [11]. The type I shunt, the umbilicalsystemic shunt, is the direct drainage of the UV into the systemic circulation. In type II shunt (ductus venosussystemic shunt) and for this type of shunt, the UV, PV and DV are intact but the DV connects to the inferior vena cava (IVC) below the pre-diaphragmatic infun- 
dibulum or the DV drains into the hepatic vein. Type III shunt, (portal-systemic shunt) is further divided into two subtypes: IIIa, i.e., the intrahepatic portal-systemic shunt (IHPSS), for which, a communication between the intrahepatic portal venous system and hepatic veins occurs; and IIIb, i.e., the extrahepatic portal-systemic shunt (EHPSS), which is defined as a shunt between the portal system and systemic veins (IVC, iliac vein and renal vein). A more detailed description of each type of UPSVS can be viewed in the schematic diagrams created by Achiron and Kivilevitch [11]. This in-utero classification has been shown to be valuable in prognosis prediction and prenatal counseling $[11,12]$. To date, different postnatal PSS categories have been used primarily for directing the surgical repair of PSS in a largely pediatric population $[13,14]$, which, however, are believed unsuitable for prenatal analysis of PSS [11]. Fetal PSS is the most common shunt seen in our clinical practice. Therefore, the aim of this study is to use the in-utero UPSVS classification to analyze the ultrasonographic characteristics and the outcome of fetal PSS, which has been, until now, poorly documented.

\section{Material and methods}

This study was approved by the Research Ethics Committee of our institution (approval No. 2017049). The Research Ethics Committee waived informed consent as the study is retrospective.

All cases of type III UPSVS diagnosed at our department from April 2016 to December 2020 were included in the present study. The demographic data, ultrasonographic findings, pregnancy outcome and follow-up results were collected and analyzed following the in-utero UPSVS classification [11].

For regular fetal monitoring, the standard 2D procedure was conducted using the Philips iU22 ultrasound system with a C5-1 transducer. When portal-systemic venous abnormalities were suspected, a 3-plane scan was further performed using the iU22 with a C5-1 transducer or the EPIQ7 with a C5-1 or an eL18-4 transducer. The 3 planes are: the transverse abdominal plane; the ventral or lateral transverse plane; and the longitudinal anteroposterior plane $[12,15]$. At the transverse abdominal plane, malformations of following structures can be detected: UV, portal sinus, main portal vein, left portal vein, anterior right portal vein, posterior right portal vein and splenic vein [15]. The ventral or lateral transverse plane scan can identify anomalies in the right, middle and left hepatic veins and IVC [15]. The longitudinal anteroposterior plane scan can discover abnormal UV, DV, left hepatic vein and IVC [15]. Any shunt between the intrahe- patic portal venous system and hepatic veins spotted by the 3-plane scan was diagnosed as type IIIa UPSVS and any shunt between the portal system and systemic veins (IVC, iliac vein and renal vein) identified by the 3-plane scan was diagnosed as type IIIb UPSVS [11].

Follow-ups were carried out 1 month, 3-6 months and 12 months post discharge, and once every year thereafter. Rearrangements were made for missing appointments. Abdominal ultrasound examination was performed to check whether the shunt was spontaneously closed or not.

\section{Statistical analysis}

The student's $t$ test was used to compare the age of patients in type IIIa and type IIIb groups. The chi-square test was performed to compare the incidence of associated anomalies and the rate of termination of pregnancy between type IIIa and type IIIb patients. All statistical analyses were done using the GraphPad Prism 8 (GraphPad Software Inc., San Diego, CA, USA). $\mathrm{p}<0.05$ was considered statistically significant.

\section{Results}

A total of 17 patients with type III UPSVS, which include 12 cases of type IIIa and 5 cases of type IIIb, were identified. The maternal age was $30.1 \pm 5.5$ years and the gestation age at diagnosis was $33.0 \pm 4.5$ weeks. There was no significant age difference between mothers

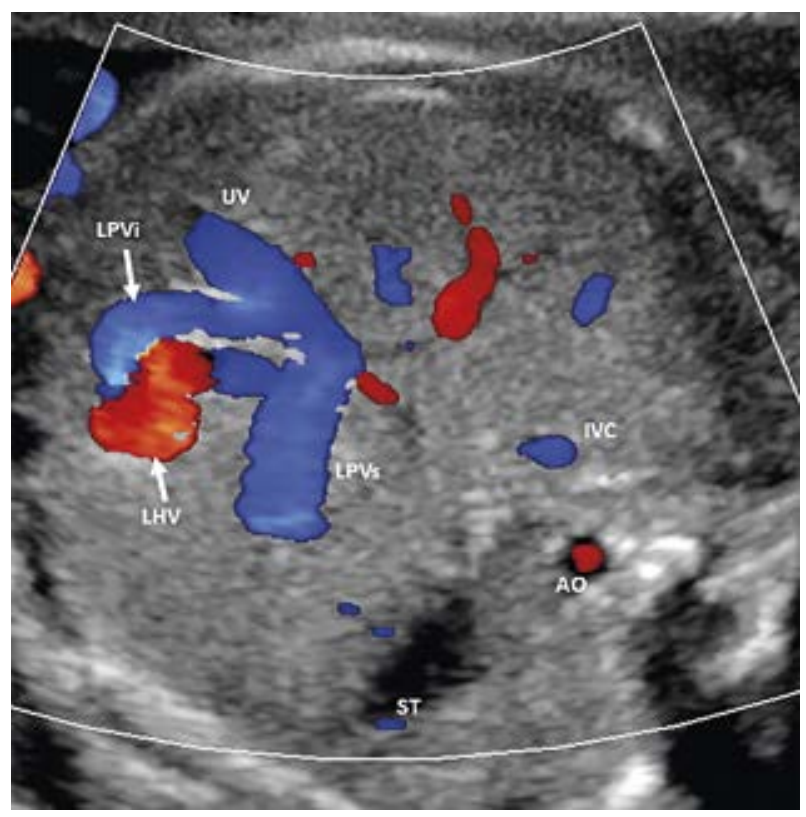

Fig 1. Representative ultrasonography of type IIIa UPSVS. A shunt between the inferior left portal vein (LPVi) and the left hepatic vein (LHV) is shown in this figure. UV: umbilical vein; LPVs: superior left portal vein; IVC: inferior vena cava; AO: aorta; and ST: stomach. 
Table I. Demographic information, shunt characteristics and birth outcome.

\begin{tabular}{lllll}
\hline Case & GA & Shunt characteristics & Associated anomalies & Outcome \\
\hline 1 & 36 & LPVi-LHV (IIIa) & None & FTB \\
2 & 32 & LPVi-LHV, LPV-LHV (IIIa) & Splenomegaly, CTR $\uparrow$ & FTB \\
3 & 28 & LPVi-MHV (IIIa) & None & FTB \\
4 & 33 & LPVi-LHV (IIIa) & IUGR & at 35w \\
5 & 32 & LPVi-LHV (IIIa) & IUGR & TOP \\
6 & 25 & LPVi-LHV, LPV-MHV (IIIa) & IUGR & TOP \\
7 & 34 & LPVi-LHV (IIIa) & IUGR \\
8 & 28 & LPVi-LHV (IIIa) & PH & C-section at 40w \\
9 & 38 & LPVi-LHV (IIIa) & IUGR, CTR $\uparrow$ & TOP \\
10 & 35 & LPVi-LHV, LPVs-LHV (IIIa) & IUGR, splenomegaly \\
11 & 39 & LPVi-LHV (IIIa) & None & FTB \\
12 & 36 & LPVi-LHV (IIIa) & IUGR & C-section at 39w \\
13 & 28 & MPV-UV, UV-IVC (IIIb and Type I) & CPC, PH, HSM, RHE & TOP \\
14 & 28 & SplV-IVC, SMV-IVC, UV-IVC (IIIb and Type I) & Multiple IHC & TOP \\
15 & 30 & MPV-IVC (IIIb) & None & C-section at 38w \\
16 & 34 & RPV-IVC (IIIb) & IUGR, Strephenopodia & TOP \\
17 & 40 & PS-IVC, LPVs-LHV, LPVm-MHV, ARPV-RHV, ARPV-MHV, & RHE, PH & FTB \\
\hline
\end{tabular}

GA: gestational age (weeks) at diagnosis; LPV: left portal vein; LPVi: inferior LPV; LHV: left hepatic vein; MHV: middle hepatic vein; MPV: main portal vein; IVC: inferior vena cava; UV: umbilical vein; PS: portal sinus; LPVs: superior LPV; LPVm: medial LPV; RPV: right portal vein; ARPV: anterior right portal vein; PRPV: posterior right portal vein; RHV: right hepatic vein; SplV: splenic vein; SMV: superior mesenteric vein; IUGR: intrauterine growth retardation; PH: polyhydramnios; CTR: cardiothoracic ratio; HSM: hepatosplenomegaly; CPC: choroid plexus cysts; IHC: intrahepatic calcifications; RHE: right heart enlargement; TOP: termination of pregnancy; FTB: full-term birth; and PB: premature birth.

with fetus with type IIIa and type IIIb (30.8 \pm 5.1 years vs. $32.6 \pm 7.9$ years, $\mathrm{p}=0.65)$. Ultrasonographic findings of the shunt in each case are presented in Table I.

All the 12 type IIIa cases had a shunt between the inferior left portal vein (LPVi) and the left hepatic vein (LHV) and a representative sonography showing such a shunt is presented in figure 1 (case 1). Three type IIIb cases had a combination of another shunt ( 2 with type I and one with type IIIa) (Table I). The ultrasonographic features of the case 17 that had type IIIb combined with type IIIa are presented in figure 2.

Several associated anomalies were detected, the most-common one being intrauterine growth restriction (IUGR) followed by polyhydramnios and increased cardiothoracic ratio. There was no significant difference in the incidence of associated anomalies between type IIIa and type IIIb patients $(\mathrm{p}=0.82)$. Prenatal karyotyping was done in 2 cases (cases 12 and 13), which showed that

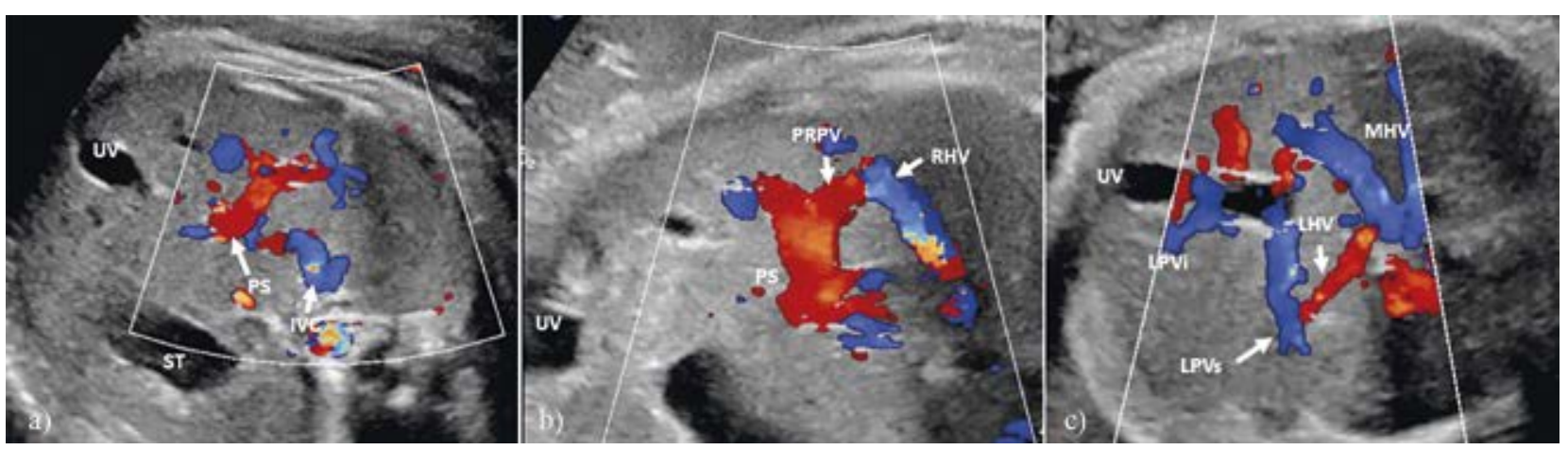

Fig 2. Representative ultrasonography of type IIIa combined with type IIIb UPSVS: a) a communication between the portal sinus (PS) and inferior vena cava (IVC) was detected; b) a shunt between the posterior right portal vein (PRPV) and the right hepatic vein (RHV) was observed; c) shunt between superior left portal vein (LPVs) and left hepatic vein (LHV). UV: umbilical vein; ST: stomach; LPVi: inferior left portal vein; and MHV: middle hepatic vein. 
case 13 had trisomy 21 while the other was normal. Termination of pregnancy (TOP) occurred in 4 cases of type IIIa as requested by the parents and 3 cases of type IIIb due to the lack of intrahepatic portal vein system. Statistical analysis showed that the rate of TOP in the two groups was not substantially different $(\mathrm{p}=0.31)$. Among the 10 survivors, case 3 had surgery 12 days post birth to close the shunt and case 17 had surgery 4 months post birth to close multiple right portal vein-right hepatic vein shunts, while the rest did not undergo any surgical procedure. The follow-up time ranged from 1 to 32 months. Ultrasonography showed spontaneous complete closure in 4 cases and spontaneous partial closure in 1 case (case 17: the LPVm-MHV shunt was closed while the LPVsLHV shunt was not), while the shunt in 4 cases were not closed during the follow-up period (Table II).

\section{Discussion}

Postnatal PSS categories have been proposed with the aim to provide criteria for surgical repair of PSS in largely pediatric patients $[13,14]$. However, these postnatal classifications refer only to the survivors, do not take into account the unique feature of the fetal UV-PVDV structure, lacking two essential components of the fetal venous complex, i.e, the UV and the DV and are therefore believed unsuitable for the prenatal analysis of portal-systemic venous anomalies. In view of this, Achiron and Kivilevitch proposed an in-utero classification for fetal UPSVS and showed its value in the prenatal analysis of UV-PV-DV abnormalities for prognosis prediction and prenatal counselling [11]. In the present study, we applied the in-utero classification to analyze the ultrasonographic characteristics of 17 cases of fetal type III UPSVS and reported the following findings: 1) the majority of cases had type IIIa UPSVS; 2) type IIIb in combination with type I UPSVS was associated with poor development of intrahepatic portal vein system, leading to dismal outcome; 3 ) approximately half of the cases (8/17) had IUGR; and 4) spontaneous shunt closure (including complete and partial) occurred in half of the survivors (5/10) during a period of 1-16 months.

Our series is the largest analyzed by the UPSVS classification. Using the UPSVS classification. Achiron et al retrospectively analyzed 16 cases of prenatally diagnosed type III shunts and showed that $75 \%(12 / 16)$ had type IIIa shunts [11], which is in line with our finding. More recently, Francois et al described the application of the Park classification for the prenatal analysis of 12 cases of intrahepatic PSS and revealed that only 3 cases had IUGR [5]. Of note, the Park classification was proposed for the postnatal analysis of PSS [16] and, by fur-
Table II. Shunt treatment and follow-up time and results.

\begin{tabular}{llll}
\hline Case & $\begin{array}{l}\text { Shunt } \\
\text { treatment }\end{array}$ & $\begin{array}{l}\text { Follow } \\
\text { time } \\
\text { (months) }\end{array}$ & Ultrasonographic findings \\
\hline 1 & None & 32 & Spontaneous closure at $16 \mathrm{~m}$ \\
2 & None & 24 & Spontaneous closure at $3 \mathrm{~m}$ \\
3 & SC at 12d & 22 & SC \\
4 & None & 17 & Spontaneous closure at $5 \mathrm{~m}$ \\
5 & None & 15 & Shunt not closed \\
8 & None & 9 & Shunt not closed \\
11 & None & 2 & Shunt not closed \\
12 & None & 1 & Spontaneous closure at $1 \mathrm{~m}$ \\
15 & None & 1 & Shunt not closed \\
17 & SC for & 10 & LPVs-LHV shunt not closed \\
& multiple & & at 7 m \\
& $\begin{array}{l}\text { RPV-RHV } \\
\text { shunts }\end{array}$ & & \\
& at 4 m & & \\
\hline
\end{tabular}

SC: surgical closure; RPV: right portal vein; RHV: right hepatic vein; LPVs: superior left portal vein; and LHV: left hepatic vein; $\mathrm{m}$ : months

ther review of the 12 cases reported by Francois et al, we found 2 cases belong to the type I UPSVS according to the Achiron and Kivilevitch classification.

It has been shown that patients with type I and II UPSVS have a high incidence of trisomy $21[11,17]$. In contrast, none of the 16 type III cases had aneuploidy [11]. We identified one case with trisomy 21 . Of note, this case had combined type I UPSVS that has been reported to be associated with trisomy 21 [17]. The genetic testing rate was low in our series and solid data that support genetic testing for patients with type III UPSVS are still lacking. Nevertheless, if patients have type III UPSVS combined with type I or type II UPSVS, genetic testing may be necessary.

In our series, TOP in 4 type IIIa cases was pursued by the parents; TOP in 3 type IIIb cases (60\%) was determined by the lack of intrahepatic portal vein system. A previous study showed that 2 out of a total of 4 type IIIb cases $(50 \%)$ underwent TOP due to the poor development of the intrahepatic portal vein system [11], in line with our findings. However, live birth was found to occur in all 12 type IIIa cases by Achiron and Kivilevitch [11], which is in contrast to our results. However, caution should be taken in the interpretation of our data, as TOP in all 4 type IIIa cases was not dictated by the nature of the shunt but rather pursued by the parents, which artificially increased the TOP rate. Additionally, the sample size is small, which may limit the statistical power.

Few authors have reported spontaneous postnatal closure of type III shunts [6]. We observed spontaneous complete closure in 4 cases. Han et al retrospectively 
studied 6 fetuses with shunts between portal and hepatic vein systems that are in line with the definition of the type IIIa shunt under the Achiron and Kivilevitch classification. Their follow up results showed 5 cases had spontaneous closure at approximately 1 year after birth [6].

\section{Conclusion}

This is the largest series of Type III shunt prenatally analyzed by ultrasonography using the new in-utero UPSVS classification. We conclude that the majority of type III UPSVS cases belong to type IIIa with good outcome and the lack of integrate intrahepatic portal vein system is the main reason for TOP in type IIIb cases. These data suggest the UPSVS classification is a useful tool for prognosis prediction of fetal PSS and prenatal counseling.

Acknowledgements: This study was supported by the Provincial Key Research and Development Fund of Shandong Province, China (Grant \#: 2015GSF118081 and 2016GSF201141).

\section{Conflict of interest: none.}

\section{References}

1. Sokollik C, Bandsma RH, Gana JC, van den Heuvel M, Ling SC. Congenital portosystemic shunt: characterization of a multisystem disease. J Pediatr Gastroenterol Nutr 2013;56:675-681.

2. Franchi-Abella S, Branchereau S, Lambert V, et al. Complications of congenital portosystemic shunts in children: therapeutic options and outcomes. J Pediatr Gastroenterol Nutr 2010;51:322-330.

3. Chocarro G, Amesty MV, Encinas JL, et al. Congenital portosystemic shunts: clinic heterogeneity requires an individual management of the patient. Eur J Pediatr Surg 2016;26:74-80.

4. Yagel S, Kivilevitch Z, Cohen SM, et al. The fetal venous system, Part II: ultrasound evaluation of the fetus with congenital venous system malformation or developing circulatory compromise. Ultrasound Obstet Gynecol 2010;36:93111.
5. Francois B, Gottrand F, Lachaux A, Boyer C, Benoit B, De Smet S. Outcome of intrahepatic portosystemic shunt diagnosed prenatally. Eur J Pediatr 2017;176:1613-1618.

6. Han BH, Park SB, Song MJ, et al. Congenital portosystemic shunts: prenatal manifestations with postnatal confirmation and follow-up. J Ultrasound Med 2013;32:45-52.

7. Delle Chiaie L, Neuberger P, Von Kalle T. Congenital intrahepatic portosystemic shunt: prenatal diagnosis and possible influence on fetal growth. Ultrasound Obstet Gynecol 2008:32:233-235.

8. Gorincour G, Droulle P, Guibaud L. Prenatal diagnosis of umbilicoportosystemic shunts: report of 11 cases and review of the literature. AJR Am J Roentgenol 2005;184:163-168.

9. Francois B, Lachaux A, Gottrand F, De Smet S. Prenatally diagnosed congenital portosystemic shunts. J Matern Fetal Neonatal Med 2018;31:1364-1368.

10. Yagel S, Kivilevitch Z, Cohen SM, et al. The fetal venous system, part I: normal embryology, anatomy, hemodynamics, ultrasound evaluation and Doppler investigation. Ultrasound Obstet Gynecol 2010;35:741-750.

11. Achiron R, Kivilevitch Z. Fetal umbilical-portal-systemic venous shunt: in-utero classification and clinical significance. Ultrasound Obstet Gynecol 2016;47:739-747.

12. Wu H, Tao G, Cong X, et al. Prenatal sonographic characteristics and postnatal outcomes of umbilical-portal-systemic venous shunts under the new in-utero classification: A retrospective study. Medicine (Baltimore) 2019;98:e14125.

13. Blanc T, Guerin F, Franchi-Abella S, et al. Congenital portosystemic shunts in children: a new anatomical classification correlated with surgical strategy. Ann Surg 2014;260:188-198.

14. Matsuura T, Takahashi Y, Yanagi Y, et al. Surgical strategy according to the anatomical types of congenital portosystemic shunts in children. J Pediatr Surg 2016;51:2099-2104.

15. Yagel S, Cohen SM, Valsky DV, Shen O, Lipschuetz M, Messing B. Systematic examination of the fetal abdominal precordial veins: a cohort study. Ultrasound Obstet Gynecol 2015;45:578-583.

16. Park JH, Cha SH, Han JK, Han MC. Intrahepatic portosystemic venous shunt. AJR Am J Roentgenol 1990;155:527528.

17. Dong X, Wu H, Zhu L, et al. Prenatal ultrasound analysis of umbilical-portal-systemic venous shunts concurrent with trisomy 21. J Ultrasound Med 2020. doi:10.1002/ jum.15507. 